\title{
ON A PRIORI ERROR ANALYSIS OF FULLY DISCRETE HETEROGENEOUS MULTISCALE FEM*
}

\author{
ASSYR ABDULLE ${ }^{\dagger}$
}

\begin{abstract}
Heterogeneous multiscale methods have been introduced by E and Engquist [Commun. Math. Sci., 1 (2003), pp. 87-132] as a methodology for the numerical computation of problems with multiple scales. Analyses of the methods for various homogenization problems have been done by several authors. These results were obtained under the assumption that the microscopic models (the cell problems in the homogenization context) are analytically given. For numerical computations, these microscopic models have to be solved numerically. Therefore, it is important to analyze the error transmitted on the macroscale by discretizing the fine scale. We give in this paper $H^{1}$ and $L^{2}$ a priori estimates of the fully discrete heterogeneous multiscale finite element method. Numerical experiments confirm that the obtained a priori estimates are sharp.
\end{abstract}

Key words. multiscale method, heterogeneous finite element method, elliptic homogenization

AMS subject classifications. 65N30, 74Q05, 74Q15, 74Q20, 39A12

DOI. $10.1137 / 040607137$

1. Introduction. The heterogeneous multiscale methods (HMM) introduced in [6] are a general framework for the numerical modeling of problems with multiple scales. For homogenization problems in a finite element (FE) context, this method discretizes the physical problem directly by a "macroscopic finite element method (FEM)" model. The fine scale of the problem is accounted for in the element stiffness matrix calculations by solving either a unit-cell problem or a problem on a patch with a fixed, i.e., scale-independent, number of unit cells. These problems will be referred to as microproblems. So far, the study of the accuracy properties in HMM has been done assuming that the fine-scale problems were analytically given [6], [1], [5], [2], [12]. Other types of multiscale methods for homogenization problems have been given in [9], [15], [11], and [8]. We note that in [11] macro- and microerrors were first separated and quantitatively estimated, although not in the HMM context and for unbounded domains. The analysis in [11] was also restricted to the case where the diffusion tensor $a^{\varepsilon}(x)=a(x / \varepsilon)$ does not depend on the macrovariable, and it cannot be easily generalized.

Recently, there have been many attempts to combine microscopic and macroscopic models for the solution of multiscale or even multiphysics problems (see, for example, [7] for a discussion on these topics). The influences of the error of a micromodel at a macroscale is an important question in multiscale computations but is, in general, difficult to address. To the best of our knowledge, this paper gives the first result of such an analysis in the HMM context.

Within the framework introduced by E, Ming, and Zhang [5] we give several error estimates for the macroscopic solutions, when the microproblems are discretized by a numerical method. These estimates include $H^{1}$ and $L^{2}$ error estimates between the solution of the finite element heterogeneous multiscale method (FE-HMM) and

\footnotetext{
*Received by the editors April 21, 2004; accepted for publication (in revised form) December 9, 2004; published electronically June 27,2005 . This work was partially supported by the Swiss National Foundation under grant 200021-103863/1.

http://www.siam.org/journals/mms/4-2/60713.html

${ }^{\dagger}$ Department of Mathematics, University of Basel, Rheinsprung 21, CH-4051 Basel, Switzerland (assyr.abdulle@unibas.ch).
} 
the solution of the homogenized problem (Theorem 3.6), $H^{1}$ and $L^{2}$ error estimates between the solution of the FE-HMM and the $L^{2}$ projection of the microscopic solution of the elliptic problem (Theorem 3.9), and, finally, $H^{1}$ error estimates between the reconstructed fine-scale solution of the FE-HMM and the microscopic solution of the elliptic problem (Theorem 3.11). Numerical experiments confirm that the derived estimates are sharp.

Our analysis shows, for periodic elliptic homogenization problems, that the macroand the micromeshes have to be refined simultaneously. This may explain the inconsistency observed in [13] between the theoretical and the numerical results for some experiments. More precisely, if $N$ denotes the number of macro degrees of freedom (DOF), and $H=\mathcal{O}\left(N^{-\frac{1}{n}}\right)$, where $n$ is the dimension of the problem, assuming that the total cost is proportional to the total DOF, the total works predicted by a semidiscrete analysis (i.e., with analytical microproblems) are $\mathcal{O}\left(N^{1 / 2}\right)$ for approximating the homogenized solution in the $L^{2}$ norm at a macrorate $\mathcal{O}\left(H^{2}\right), \mathcal{O}(N)$ for approximating the homogenized solution in the $H^{1}$ norm at a macrorate $\mathcal{O}(H)$, and $\mathcal{O}(N)$ for approximating the fine-scale solution in the $H^{1}$ norm at a macrorate $\mathcal{O}(H)$ [13], [6], [5], [2]. The fully discrete analysis, with a numerical microscopic solution, shows that the total works are $\mathcal{O}(N), \mathcal{O}\left(N^{3 / 2}\right)$, and $\mathcal{O}\left(N^{2}\right)$, respectively, for the three aforementioned cases. Furthermore, numerical experiments show that these estimates are sharp.

We recall that the FE-HMM has a robust convergence (towards the homogenized solution) in $H$ which does not depend on $\varepsilon$ [6], [2]. Finally, since each microproblem is independent, the cell problems can be solved in parallel, and, in this case, the total work is $\mathcal{O}(N)$, independently of $\varepsilon$.

For brevity of exposition, we will present the results for piecewise linear continuous FEMs in the micro- and in the macrospaces. We emphasize that our results can be extended to higher orders with the same techniques. Although the FE-HMM is applicable also in the nonperiodic setting (see section 2), our analysis is done in the periodic case, since, in this case, the analytical behavior of the true solution, as well as its homogenized limit, is well understood.

The paper is organized as follows. In section 2 we describe the FE-HMM for elliptic problems and recall known convergence results. In section 3 we give the convergence results for the fully discrete FE-HMM. Finally, in section 4, we present numerical examples which illustrate that the $H^{1}$ a priori estimates given in section 3 are sharp.

Notation. In what follows, $C>0$ denotes a generic constant, independent of $\varepsilon$, whose value can change at any occurrence but depends only on the quantities which are indicated explicitly. For $r=\left(r_{1}, \ldots, r_{n}\right) \in \mathbb{N}^{n}$, we denote $|r|=r_{1}+\cdots+r_{n}$, $D^{r}=\partial_{1}^{r_{1}} \ldots \partial_{n}^{r_{n}}$. We will consider the usual Sobolev space $H^{1}(\Omega)=\left\{u \in L^{2}(\Omega)\right.$; $\left.D^{r} u \in L^{2}(\Omega),|r| \leq 1\right\}$, with norm $\|u\|_{H^{1}(\Omega)}=\left(\sum_{|r| \leq 1}\left\|D^{r} u\right\|_{L^{2}(\Omega)}^{2}\right)^{1 / 2}$. We will also consider $H_{0}^{1}(\Omega)$ the closure of $C_{0}^{\infty}(\Omega)$ for the $\|\cdot\|_{H^{1}(\Omega)}$ norm and the spaces $W^{l, \infty}(\Omega)=\left\{u \in L^{\infty}(\Omega) ; D^{r} u \in L^{\infty}(\Omega),|r| \leq l\right\}$. Finally, we will consider $W_{p e r}^{1}(Y)=$ $\left\{v \in H_{\text {per }}^{1}(Y) ; \int_{Y} v d x=0\right\}$, where $H_{\text {per }}^{1}(Y)$ is defined as the closure of $\mathcal{C}_{\text {per }}^{\infty}(Y)$ (the subset of $\mathcal{C}^{\infty}\left(\mathbb{R}^{n}\right)$ of periodic functions in the unit cube $\left.Y=(0,1)^{n}\right)$ for the $H^{1}$ norm.

2. HMM for elliptic homogenization problems. We consider the following elliptic model problem in the domain $\Omega \subset \mathbb{R}^{n}$ :

$$
-\nabla \cdot\left(a^{\varepsilon} \nabla u^{\varepsilon}\right)=f \text { in } \Omega, \quad u^{\varepsilon}=0 \text { on } \partial \Omega,
$$


where we assume that the tensor $a^{\varepsilon}(x)=a\left(x, \frac{x}{\varepsilon}\right)=a(x, y)$ is symmetric, coercive, and periodic with respect to each component of $y$ in the unit cube $Y=(0,1)^{n}$. We further assume that $f \in L^{2}(\Omega), a_{i j}(x, \cdot) \in L^{\infty}\left(\mathbb{R}^{n}\right)$, and $x \rightarrow a_{i j}(x, \cdot)$ is smooth from $\bar{\Omega} \rightarrow L^{\infty}\left(\mathbb{R}^{n}\right)$. We also assume that $\Omega$ is a convex polygon. Throughout, we add a superscript on the solution $u$ to emphasize its dependence on $\varepsilon$. It is known from homogenization theory (see, e.g., [3, Chap. 1], [14]) that $u^{\varepsilon}$ converges (usually in a weak sense) to a "homogenized solution" $u^{0}$, a solution of the homogenized problem

$$
-\nabla \cdot\left(a^{0}(x) \nabla u^{0}\right)=f(x) \in \Omega, \quad u^{0}=0 \quad \text { on } \partial \Omega,
$$

where the homogenized diffusion coefficient $a^{0}$ is a smooth matrix with coefficients given by $a_{i j}^{0}(x)=\int_{Y}\left(a_{i j}(x, y)+\sum_{k=1}^{n} a_{i k}(x, y) \frac{\partial \chi^{j}}{\partial y_{k}}(x, y)\right) d y$. Here, $\chi^{j}(x, \cdot)$ denote the solutions of the cell problems given in (2.8). Classical homogenization theory [3], [14], [10] gives

$$
\left\|u^{\varepsilon}-u^{0}\right\|_{L^{2}(\Omega)} \leq C \varepsilon
$$

Some regularity on $\chi^{j}(x, \cdot)$ is needed for this estimate (see [9, Rem. 3.3]). The assumptions we make in section 3 (see 3.9) are sufficient for estimation (2.3) to hold.

As mentioned in the introduction, we concentrate here for simplicity on piecewise linear continuous FEMs in the micro- and in the macrospaces. Therefore, let the macro FE space be defined by

$$
S_{0}^{1}\left(\Omega, \mathcal{T}_{H}\right)=\left\{u^{H} \in H_{0}^{1}(\Omega) ;\left.u^{H}\right|_{K} \in \mathcal{P}^{1}(K) \forall K \in \mathcal{T}_{H}\right\},
$$

where $\mathcal{P}^{1}(K)$ is the space of linear polynomials on the triangle $K$, and $\mathcal{T}_{H}$ is a quasiuniform triangulation of $\Omega \subset \mathbb{R}^{n}$ of shape regular triangles $K$. By "macrofinite elements" we mean that $H$, the size of the triangulation, can be larger than the length scale $\varepsilon$.

Standard a priori estimates give $\left\|u^{\varepsilon}-u^{H}\right\|_{H^{1}(\Omega)} \leq C(H / \varepsilon)\|f\|_{L^{2}(\Omega)}$, where we use the estimate $|u|_{H^{2}(\Omega)} \leq(C / \varepsilon)\|f\|_{L^{2}(\Omega)}$ for $H^{2}$-regular functions. (The factor $1 / \varepsilon$ is due to the small oscillations in $u^{\varepsilon}$; see [9, equation (4.4)].)

The FE-HMM for the elliptic homogenization problems, based on the macrospace $S_{0}^{1}\left(\Omega, \mathcal{T}_{H}\right)$, is defined by a modified macrobilinear form [6], [2],

$$
B\left(u^{H}, v^{H}\right)=\sum_{K \in \mathcal{T}_{H}} \frac{|K|}{\left|K_{\varepsilon}\right|} \int_{K_{\varepsilon}} \nabla u a\left(x_{k}, x / \varepsilon\right)(\nabla v)^{T} d x,
$$

where $K_{\varepsilon}=x_{k}+\varepsilon[-1 / 2,1 / 2]^{n}$ is a sampling subdomain centered at the barycenter $x_{k}$ of $K$ and $|K|,\left|K_{\varepsilon}\right|$ denote the measure of $K$ and $K_{\varepsilon}$, respectively, and where $u$ is the solution of the following microproblem: find $u$ such that $\left(u-u^{H}\right) \in W_{p e r}^{1}\left(K_{\varepsilon}\right)$ and

$$
\int_{K_{\varepsilon}} \nabla u a\left(x_{k}, x / \varepsilon\right)(\nabla z)^{T} d x=0 \quad \forall z \in W_{p e r}^{1}\left(K_{\varepsilon}\right) .
$$

We obtain $v$ by a similar problem replacing $u^{H}$ by $v^{H}$. It is shown in [1, sect. 2.3], [5], [2, App. A] that

$$
u=u^{H}+\varepsilon \sum_{j=1}^{n} \chi^{j}\left(x_{k}, x / \varepsilon\right) \frac{\partial u^{H}\left(x_{k}\right)}{\partial x_{j}},
$$


where $\chi^{j}\left(x_{k}, y\right), j=1, \ldots, n$, are the (unique) solutions of the cell problems

$$
\int_{Y} \nabla \chi^{j} a\left(x_{k}, y\right)(\nabla z)^{T} d y=-\int_{Y} e_{j}^{T} a\left(x_{k}, y\right)(\nabla z)^{T} d y \quad \forall z \in W_{p e r}^{1}(Y),
$$

where $Y=(0,1)^{n}$ and $\left\{e_{j}\right\}_{j=1}^{n}$ is the standard basis of $\mathbb{R}^{n}$. These latter cell problems are the standard cell problems upon which the homogenized coefficients of problem (2.1) are constructed, and (2.7), with $u^{H}$ replaced by $u^{0}$ (the homogenized solution), is known in homogenization theory as the first approximation of the solution $u^{\varepsilon}$ (see [3, Chap. 1]).

REMARK 2.1. In the definition (2.5), we collocate the slow variable of the tensor $a(x, x / \varepsilon)$ at the barycenter $x_{k}$ of $K_{\varepsilon}$ as it was done in [2]. This allows us to obtain error estimates (see (2.14)) consistent with the homogenized solution $u^{0}$.

The macrosolution of the FE-HMM is defined by the following variational problem [6], [5], [2]: find $u^{H} \in S_{0}^{1}\left(\Omega, \mathcal{T}_{H}\right)$ such that

$$
B\left(u^{H}, v^{H}\right)=\left\langle f, v^{H}\right\rangle \quad \forall v^{H} \in S_{0}^{1}\left(\Omega, \mathcal{T}_{H}\right),
$$

where $\langle\cdot, \cdot\rangle$ denotes the standard scalar product of $L^{2}$ functions. It can be shown that the bilinear form $B(\cdot, \cdot)$ is elliptic and bounded, and thus the problem (2.9) admits a unique solution. It can also be shown that

$$
B\left(u^{H}, v^{H}\right)=\sum_{K \in \mathcal{T}_{H}} \int_{K} \nabla u^{H} a^{0}\left(x_{k}\right)\left(\nabla v^{H}\right)^{T} d x,
$$

where $a_{0}$ is the homogenized tensor given in (2.2) (see [6], [2, App. A], [5]). Notice that unless problem (2.6) is analytically given, (2.9) is of semidiscrete nature, since it assumes an exact microsolver. In section 3 we will drop this assumption and consider the fully discrete problem.

The following convergence estimates have been obtained in [5] for the macrospace $S_{0}^{1}\left(\Omega, \mathcal{T}_{H}\right)$, assuming $H^{2}$ regularity for the solution $u^{0}$ of the homogenized problem (2.2) and an exact solution of (2.6):

$$
\begin{aligned}
& \left\|u^{0}-u^{H}\right\|_{H^{1}(\Omega)} \leq C(\varepsilon+H)\|f\|_{L^{2}(\Omega)}, \\
& \left\|u^{\varepsilon}-u_{p}^{\varepsilon}\right\|_{\bar{H}^{1}(\Omega)} \leq C(\sqrt{\varepsilon}+H)\|f\|_{L^{2}(\Omega)}, \\
& \left\|P u^{\varepsilon}-u^{H}\right\|_{H^{1}(\Omega)} \leq C(\varepsilon / H+H)\|f\|_{L^{2}(\Omega)},
\end{aligned}
$$

where $u^{\varepsilon}$ is the solution of problem (2.1), $u^{0}$ is the solution of the homogenized problem (2.2), and $u_{p}^{\varepsilon}$ is a reconstructed solution obtained from $u^{H}$ with fine-scale solution $\left(u-u^{H}\right)$ periodically extended on each element $K$. For the estimate (2.12), the norm $\bar{H}$ is a mesh-dependent norm since $u_{p}^{\varepsilon}$ can be discontinuous across the macroelements $K$ (see section 3 for details). Finally, $P u^{\varepsilon}$ denotes the $L^{2}$ projection of the solution. We refer the reader to [5] for the proofs of the above estimates.

If we collocate the slow variable of the tensor $a\left(x_{k}, x / \varepsilon\right)$ at the barycenter $x_{k}$ of $K_{\varepsilon}$, as done in the bilinear form (2.5), estimation (2.11) can be improved, and one can obtain (see [2, App. A])

$$
\left\|u^{0}-u^{H}\right\|_{H^{1}(\Omega)} \leq C H\|f\|_{L^{2}(\Omega)} .
$$

Compared to a standard FEM for the problem (2.1), we do not solve the fine-scale problems on the whole domain $\Omega$ but only on sampling domains $K_{\varepsilon}$, which can be chosen of the size of $\varepsilon$ in the periodic case. The fine-scale solution can be reconstructed from the known macro- and microsolutions on $K$ and $K_{\varepsilon}$, respectively. Finally, we note that the work on each sampling domain can be done in parallel. 
2.1. A remark on the nonperiodic case. The algorithm described above relies on a modified macrobilinear form, where, in place of numerical integration, we solve microscale problems on sampling domains $K_{\varepsilon}$. In the periodic case, as previously mentioned, we can choose $K_{\varepsilon}$ of size $\varepsilon^{n}$. In the general case, when $a^{\varepsilon}$ is not necessarily periodic, there are two main issues:

- $K_{\varepsilon}$ should be replaced by $K_{\delta}=x_{k}+\delta[-1 / 2,1 / 2]^{n}$, where $\delta$ is chosen such that it samples enough information of the local variations of $a^{\varepsilon}$.

- The boundary conditions of the microproblem have to be appropriately chosen.

In [5] it is proposed to use $u=u^{H}$ on $\partial K_{\delta}$ as boundary conditions for the microsolutions (2.6) solved in $H^{1}\left(K_{\delta}\right)$. With these boundary conditions the semidiscrete formulation (2.9) has been analyzed in the periodic case (although, in that case, the periodic boundary conditions (2.6) are optimal). The case of a stationary random field $a^{\varepsilon}$ has also been investigated numerically in [1] (in a finite difference context) and in [13] (in a FE context). In this latter case, the semidiscrete formulation has been partially analyzed [5]. Notice that, in this case also, periodic boundary conditions as in $(2.6)$ can still be retained.

3. Error analysis of the fully discrete FE-HMM. We estimate here the solution of the problem (2.9), when (2.6) is defined on a micro FE space. We recall that the sampling domain is defined as $K_{\varepsilon}=x_{k}+\varepsilon[-1 / 2,1 / 2]^{n}$, where $x_{k}$ is at the barycenter of the macroelement $K$, and we define $\mathcal{T}_{h}$, a quasi-uniform triangulation of the sampling domain $K_{\varepsilon}$, with mesh size $h$. We consider a micro FE space $S_{\text {per }}^{1}\left(K_{\varepsilon}, \mathcal{T}_{h}\right) \subset W_{\text {per }}^{1}\left(K_{\varepsilon}\right)$ defined by

$$
S_{\text {per }}^{1}\left(K_{\varepsilon}, \mathcal{T}_{h}\right)=\left\{u^{h} \in W_{p e r}^{1}\left(K_{\varepsilon}\right) ;\left.u^{h}\right|_{T} \in \mathcal{P}^{1}(T), T \in \mathcal{T}_{h}\right\},
$$

where $\mathcal{P}^{1}(T)$ is the space of linear polynomials on the triangle $T$.

The discrete microproblem is defined in the following way. For $u^{H} \in S_{0}^{1}\left(\Omega, \mathcal{T}_{H}\right)$, find $u^{h}$ such that $\left(u^{h}-u^{H}\right) \in S_{p e r}^{1}\left(K_{\varepsilon}, \mathcal{T}_{h}\right)$ and

$$
\int_{K_{\varepsilon}} \nabla u^{h} a\left(x_{k}, x / \varepsilon\right)\left(\nabla z^{h}\right)^{T} d x=0 \quad \forall z^{h} \in S_{p e r}^{1}\left(K_{\varepsilon}, \mathcal{T}_{h}\right) .
$$

It can be shown similarly as in [4, Chap. 6.1] that the problem (3.2) has a unique solution.

REMARK 3.1. For a practical implementation, it has been shown in [2] that periodic boundary conditions can be enforced weakly through Lagrange multipliers in nonperiodic FE spaces.

Similarly to (2.7), we have the following expression for the solution $u^{h}$ of (3.2):

$$
u^{h}=u^{H}+\varepsilon \sum_{j=1}^{n} \chi^{j, h}\left(x_{k}, x / \varepsilon\right) \frac{\partial u^{H}\left(x_{k}\right)}{\partial x_{j}},
$$

where $\chi^{j, h}\left(x_{k}, x / \varepsilon\right), j=1, \ldots, n$, are the solutions of the cell problems (2.8) in $S_{\text {per }}^{1}\left(K_{\varepsilon}, \mathcal{T}_{h}\right)$ instead of $W_{\text {per }}^{1}\left(K_{\varepsilon}\right)$. Indeed, inserting (3.3) in (3.2) and using that $\nabla u^{H}$ is constant on $K$ (since $u^{H} \in S_{0}^{1}\left(\Omega, \mathcal{T}_{H}\right)$ ) and that $\chi^{j, h}$ are the solutions of the cell problems (2.8) in $S_{p e r}^{1}\left(K_{\varepsilon}, \mathcal{T}_{h}\right)$ give the result. Notice that if $K \subset K_{\varepsilon}$, the value of $\nabla u^{H}$ is well defined since we take it at the barycenter $x_{k} \in K$. 
The fully discrete macrobilinear form is now given by

$$
\bar{B}\left(u^{H}, v^{H}\right)=\sum_{K \in \mathcal{T}_{H}} \frac{|K|}{\left|K_{\varepsilon}\right|} \int_{K_{\varepsilon}} \nabla u^{h} a\left(x_{k}, x / \varepsilon\right)\left(\nabla v^{h}\right)^{T} d x .
$$

The corresponding macro FE-HMM solution is defined by the following variational problem: find $\bar{u}^{H} \in S_{0}^{1}\left(\Omega, \mathcal{T}_{H}\right)$ such that

$$
\bar{B}\left(\bar{u}^{H}, v^{H}\right)=\left\langle f, v^{H}\right\rangle \quad \forall v^{H} \in S_{0}^{1}\left(\Omega, \mathcal{T}_{H}\right) .
$$

The proposition below shows that (3.5) is well posed and has a unique solution.

Proposition 3.2. The problem (3.5) has a unique solution which satisfies

$$
\left\|\bar{u}^{H}\right\|_{H^{1}(\Omega)} \leq C\|f\|_{L^{2}(\Omega)} .
$$

Proof. Let $v^{H} \in S_{0}^{1}\left(\Omega, \mathcal{T}_{H}\right)$. Since $v^{h}-v^{H} \in S_{\text {per }}^{1}\left(K_{\varepsilon}\right)$ and $\nabla v^{H}$ is constant over a macrotriangle $K$, we have

$$
\begin{aligned}
& \int_{K_{\varepsilon}}\left|\nabla v^{h}\right|^{2} d x=\int_{K_{\varepsilon}}\left|\nabla v^{h}-\nabla v^{H}\right|^{2} d x+\int_{K_{\varepsilon}}\left|\nabla v^{H}\right|^{2} d x, \\
& \int_{K_{\varepsilon}} \nabla v^{h} a\left(x_{k}, x / \varepsilon\right)\left(\nabla v^{h}-\nabla v^{H}\right)^{T} d x=0 .
\end{aligned}
$$

Equality (3.7) implies that $B\left(v^{H}, v^{H}\right) \geq C\left\|v^{H}\right\|_{H^{1}(K)}^{2}$, and thus the bilinear form $\bar{B}$ is coercive. Equality (3.8) implies that $\left\|\nabla v^{h}\right\|_{L^{2}\left(K_{\varepsilon}\right)} \leq C\left\|\nabla v^{H}\right\|_{L^{2}\left(K_{\varepsilon}\right)}$, and it follows that $\bar{B}$ is bounded. The existence and uniqueness of a solution $\bar{u}^{H}$ of problem (3.5), as well as (3.6), follow from the Lax-Milgram theorem.

In what follows, we assume that the solutions $\chi^{j}$ of the cell problems (2.8) satisfy $\chi^{j}\left(x_{k}, \cdot\right) \in W^{2, \infty}(Y)$. If one sets $\chi^{j}\left(x_{k}, y\right)=\chi^{j}\left(x_{k}, x / \varepsilon\right)$, then by the chain rule (assuming $\chi^{j}$ is smooth) we have

$$
\left\|D_{x}^{\alpha}\left(\chi^{j}\left(x_{k}, x / \varepsilon\right)\right)\right\|_{L^{\infty}\left(K_{\varepsilon}\right)} \leq C \varepsilon^{-|\alpha|},|\alpha| \leq 2, \alpha \in \mathbb{N}^{n} .
$$

As a preparation for the error estimate we prove the following lemma.

Lemma 3.3. Suppose that the solutions of the cell problem (2.8) satisfy (3.9). Then the following estimation holds:

$$
\left|\bar{B}\left(v^{H}, w^{H}\right)-B\left(v^{H}, w^{H}\right)\right| \leq C\left(\frac{h}{\varepsilon}\right)^{2}\left\|\nabla v^{H}\right\|_{L^{2}(\Omega)}\left\|\nabla w^{H}\right\|_{L^{2}(\Omega)},
$$

where $v^{H}, w^{H} \in S_{0}^{1}\left(\Omega, \mathcal{T}_{H}\right), h$ is the mesh size of the micro FEM (3.1), and $\bar{B}$ and $B$ are defined in (3.4) and (2.5), respectively.

Proof. Using the definitions of (3.4) and (2.5) we have

$$
\begin{gathered}
\left|B\left(v^{H}, w^{H}\right)-\bar{B}\left(v^{H}, w^{H}\right)\right| \\
=\left|\sum_{K \in \mathcal{T}} \frac{|K|}{\left|K_{\varepsilon}\right|}\left(\int_{K_{\varepsilon}} \nabla v a\left(x_{k}, x / \varepsilon\right)(\nabla w)^{T} d x-\int_{K_{\varepsilon}} \nabla v^{h} a\left(x_{k}, x / \varepsilon\right)\left(\nabla w^{h}\right)^{T} d x\right)\right| \\
=\mid \sum_{K \in \mathcal{T}} \frac{|K|}{\left|K_{\varepsilon}\right|}\left(\int_{K_{\varepsilon}} \nabla\left(v-v^{h}\right) a\left(x_{k}, x / \varepsilon\right)(\nabla w)^{T} d x\right. \\
\left.\quad-\int_{K_{\varepsilon}} \nabla v^{h} a\left(x_{k}, x / \varepsilon\right)\left(\nabla\left(w^{h}-w\right)\right)^{T} d x\right) \mid
\end{gathered}
$$


We observe that the first member of the last line of (3.11) is zero since $\left(v-v^{h}\right) \in$ $W_{p e r}^{1}\left(K_{\varepsilon}\right)$. Using the same argument and replacing $v^{h}$ by $v^{h}-v$ in the second expression, we have

$$
\begin{aligned}
& \left|\sum_{K \in \mathcal{T}} \frac{|K|}{\left|K_{\varepsilon}\right|} \int_{K_{\varepsilon}} \nabla\left(v^{h}-v\right) a\left(x_{k}, x / \varepsilon\right)\left(\nabla\left(w^{h}-w\right)\right)^{T} d x\right| \\
& \leq C \sum_{K \in \mathcal{T}} \frac{|K|}{\left|K_{\varepsilon}\right|}\left\|\nabla v^{h}-\nabla v\right\|_{L^{2}\left(K^{\varepsilon}\right)}\left\|\nabla w^{h}-\nabla w\right\|_{L^{2}\left(K^{\varepsilon}\right)},
\end{aligned}
$$

where we used that the bilinear form is bounded. It remains to estimate

$$
\begin{aligned}
& \left\|\nabla v^{h}-\nabla v\right\|_{L^{2}\left(K_{\varepsilon}\right)}=\left\|\varepsilon \sum_{j=1}^{n} \nabla\left(\chi^{j, h}\left(x_{k}, x / \varepsilon\right)-\chi^{j}\left(x_{k}, x / \varepsilon\right)\right) \frac{\partial v^{H}}{\partial x_{j}}\right\|_{L^{2}\left(K_{\varepsilon}\right)} \\
& \leq C \sqrt{\nabla v^{H} \nabla v^{H}} \max _{j}\left\|\varepsilon \nabla\left(\chi^{j, h}-\chi^{j}\right)\right\|_{L^{2}\left(K_{\varepsilon}\right)} \\
& \leq C h \sqrt{\nabla v^{H} \nabla v^{H}} \varepsilon \max _{j}\left|\chi^{j}\right|_{W^{2, \infty}\left(K_{\varepsilon}\right)} \sqrt{\left|K_{\varepsilon}\right|} \leq C(h / \varepsilon) \sqrt{\nabla v^{H} \nabla v^{H}} \sqrt{\left|K_{\varepsilon}\right|},
\end{aligned}
$$

where we used the standard result for $H^{1}$ error estimates (with $H^{2}$ regularity of $\chi^{j}$ ), and the assumption (3.9). Since $\nabla v^{H}$ is constant, we have

$$
\sqrt{|K| /\left|K_{\varepsilon}\right|} \sqrt{\nabla v^{H} \nabla v^{H}} \sqrt{\left|K_{\varepsilon}\right|}=\left\|\nabla v^{H}\right\|_{L^{2}(K)} .
$$

The second term of (3.13), $\left\|\nabla w^{h}-\nabla w\right\|_{L^{2}\left(K_{\varepsilon}\right)}$, is treated similarly. Finally, summing up over $K \in \mathcal{T}$ we find that (3.13) is bounded by $C\left(\frac{h}{\varepsilon}\right)^{2}\left\|\nabla v^{H}\right\|_{L^{2}(\Omega)}\left\|\nabla w^{H}\right\|_{L^{2}(\Omega)}$, and the proof is complete.

REMARK 3.4. If we denote $M=\operatorname{dim} S_{p e r}^{1}\left(K_{\varepsilon}\right)$, then the mesh size of the micro FE space (3.1) on $K_{\varepsilon}$ (of measure $\left|K_{\varepsilon}\right|=\varepsilon^{n}$ ) is given by $h \simeq \varepsilon M^{-\frac{1}{n}}$. Therefore, the quantity $h / \varepsilon$ in (3.10) is independent of $\varepsilon$ for the micro FEM applied to the cell problems (3.2), and we express it as $M^{-\frac{1}{n}}$, which emphasizes that it depends only on the DOF of $S_{p e r}^{1}\left(K_{\varepsilon}\right)$.

We estimate next the error introduced in the macrosolution $u^{H}$, when the microproblem is given by (3.2).

Proposition 3.5. Let $u^{H}, \bar{u}^{H}$ be the solutions of problem (2.9) and (3.5), respectively, and suppose that the assumptions of Lemma 3.3 hold. Then

$$
\left\|u^{H}-\bar{u}^{H}\right\|_{H^{1}(\Omega)} \leq C M^{-\frac{2}{n}}\|f\|_{L^{2}(\Omega)},
$$

where $M$ is the dimension of the micro FE space $S_{p e r}^{1}\left(K_{\varepsilon}\right)$ defined in (3.1).

Proof. Denoting by $w^{H}=u^{H}-\bar{u}^{H}$, we have

$$
\alpha\left\|u^{H}-\bar{u}^{H}\right\|_{H^{1}(\Omega)}^{2} \leq B\left(u^{H}-\bar{u}^{H}, w^{H}\right)=\bar{B}\left(\bar{u}^{H}, w^{H}\right)-B\left(\bar{u}^{H}, w^{H}\right),
$$

and thus

$$
\left\|u^{H}-\bar{u}^{H}\right\|_{H^{1}(\Omega)} \leq \frac{1}{\alpha} \frac{\left|\bar{B}\left(\bar{u}^{H}, w^{H}\right)-B\left(\bar{u}^{H}, w^{H}\right)\right|}{\left\|w^{H}\right\|_{H^{1}(\Omega)}} .
$$

Using Lemma 3.3 and (3.6) leads to the result. 
We can now give the error estimate between the homogenized solution and the solution of the fully discretized FE-HMM.

THEOREM 3.6. Let $u^{0}$ be the solution of the homogenized problem (2.2), and assume $u^{0}$ is $H^{2}$-regular. Let $\bar{u}^{H}$ be the solution of problem (3.5), and suppose that the assumptions of Lemma 3.3 hold. Then

$$
\left\|u^{0}-\bar{u}^{H}\right\|_{H^{1}(\Omega)} \leq C\left(H+M^{-\frac{2}{n}}\right)\|f\|_{L^{2}(\Omega)},
$$

where $H$ is the size of the triangulation of the macro FE space (2.4) and $M$ is the dimension of the micro FE space $S_{p e r}^{1}\left(K_{\varepsilon}\right)$ defined in (3.1).

Proof. Using (3.15) and (2.14) gives the result.

Corollary 3.7. Suppose that the assumptions of Theorem 3.6 hold. Then we have the following estimates:

$$
\begin{aligned}
& \left\|u^{0}-\bar{u}^{H}\right\|_{L^{2}(\Omega)} \leq C\left(H^{2}+M^{-\frac{2}{n}}\right)\|f\|_{L^{2}(\Omega)}, \\
& \left\|u^{\varepsilon}-\bar{u}^{H}\right\|_{L^{2}(\Omega)} \leq C\left(H^{2}+\varepsilon+M^{-\frac{2}{n}}\right)\|f\|_{L^{2}(\Omega)} .
\end{aligned}
$$

Proof. Estimation (3.19) follows from estimation $\left\|u^{0}-u^{H}\right\|_{L^{2}(\Omega)} \leq C H^{2}\|f\|_{L^{2}(\Omega)}$ for the solution of (2.9) (see [2, App. A]), estimation (3.15), and the triangle inequality. Estimation (3.20) follows from (2.3), (3.19), and the triangle inequality.

REMARK 3.8. Numerical examples given in section 4 indicate that the estimations (3.18), (3.20), and (3.19) are sharp.

We next derive convergence results for the $L^{2}$ projection of the solution of (2.1). For $u \in H^{1}(\Omega)$ we define $P u \in S_{0}^{1}\left(\Omega, \mathcal{T}_{H}\right)$ as the unique solution of the problem

$$
\left\langle P u, v^{H}\right\rangle=\left\langle u, v^{H}\right\rangle \quad \forall v^{H} \in S_{0}^{1}\left(\Omega, \mathcal{T}_{H}\right) .
$$

THEOREM 3.9. Let $P u^{\varepsilon}$ be the solution of $(2.1)$ projected on $S_{0}^{1}\left(\Omega, \mathcal{T}_{H}\right)$ by (3.21), and let $\bar{u}^{H}$ be the solution of problem (3.5). Suppose that the assumptions of Lemma 3.3 hold and that $u^{0}$, the solution of (2.2), is $H^{2}$-regular. Then

$$
\left\|P u^{\varepsilon}-\bar{u}^{H}\right\|_{H^{1}(\Omega)} \leq C\left(\frac{\varepsilon}{H}+H+M^{-\frac{2}{n}}\right)\|f\|_{L^{2}(\Omega)},
$$

where $H$ is the size of the triangulation of the macro FE space (2.4) and $M$ is the dimension of the micro FE space $S_{\text {per }}^{1}\left(K_{\varepsilon}\right)$ defined in (3.1).

Proof. Using (3.15), (2.13), and the triangle inequality gives the result. (Notice that the $H^{2}$ regularity of $u^{0}$ is used in (2.13).)

Corollary 3.10. Suppose that the assumptions of Theorem 3.9 hold. Then

$$
\left\|P u^{\varepsilon}-\bar{u}^{H}\right\|_{L^{2}(\Omega)} \leq C\left(\varepsilon+H^{2}+M^{-\frac{2}{n}}\right)\|f\|_{L^{2}(\Omega)} .
$$

Proof. The estimation follows from $\left\|P u^{\varepsilon}-u^{H}\right\|_{L^{2}(\Omega)} \leq C\left(\varepsilon+H^{2}\right)$ (see [5]), estimation (3.15), and the triangle inequality.

Finally, we consider a procedure to retrieve the microscopic information. Following [16] (see also [1], [5]), we define $\bar{u}_{p}^{\varepsilon}$ by

$$
\bar{u}_{p}^{\varepsilon}(x)=\bar{u}^{H}(x)+\left.\left(u^{h}(x)-\bar{u}^{H}(x)\right)\right|_{K} ^{P} \quad \text { for } x \in K \in \mathcal{T}_{H},
$$

where $\left.\right|_{K} ^{P}$ denotes the periodic extension of the fine-scale solution $\left(u^{h}-\bar{u}^{H}\right)$, available in $K_{\varepsilon}$, on each element $K$. This extension is defined for a function $w \in H^{1}\left(K_{\varepsilon}\right)$ by

$$
w_{p}(x+\varepsilon l)=w(x) \quad \forall l=\left(l_{l}, \ldots, l_{n}\right) \in \mathbb{Z}^{n}, \forall x \in K_{\varepsilon} \quad \text { such that } x+\varepsilon l \in K .
$$


The function $\bar{u}_{p}^{\varepsilon}$, which can still be expressed by (3.3) (but now for the whole macroelement $K$ ), defines a fully discrete fine-scale approximation of the fine-scale solution $u^{\varepsilon}$ of problem (2.1). Since $\bar{u}_{p}^{\varepsilon}$ can be discontinuous across the macroelements $K$, we define a broken $H^{1}$ norm by

$$
\|u\|_{\bar{H}^{1}(\Omega)}:=\left(\sum_{K \in \mathcal{T}_{H}}\|\nabla u\|_{L^{2}(K)}^{2}\right)^{\frac{1}{2}} .
$$

THEOREM 3.11. Let $\bar{u}_{p}^{\varepsilon}$ be defined by (3.24), and let $u^{\varepsilon}$ be the solution of (2.1). Suppose that the assumptions of Lemma 3.3 hold. Then

$$
\left\|u^{\varepsilon}-\bar{u}_{p}^{\varepsilon}\right\|_{\bar{H}^{1}(\Omega)} \leq C\left(\sqrt{\varepsilon}+H+M^{-\frac{1}{n}}\right)\|f\|_{L^{2}(\Omega)},
$$

where $H$ is the size of the triangulation of the macro FE space (2.4), $M$ is the dimension of the micro FE space $S_{\text {per }}^{1}\left(K_{\varepsilon}\right)$ defined in (3.1), and $\bar{H}^{1}$ is the broken norm defined in (3.25).

Proof. We consider $u_{p}^{\varepsilon}=u^{H}+\left(u-u^{H}\right)_{K}^{P}$, the function similar to (3.24) but with the exact cell problems (see (2.7)). A direct calculation, invoking (3.3) and (2.7), gives

$$
\begin{aligned}
& \nabla u_{p}^{\varepsilon}=\nabla u^{H}+\sum_{j=1}^{n} \nabla\left(\varepsilon \chi^{j}\left(x_{k}, x / \varepsilon\right)\right) \frac{\partial u^{H}}{\partial x_{j}}, \\
& \nabla \bar{u}_{p}^{\varepsilon}=\nabla \bar{u}^{H}+\sum_{j=1}^{n} \nabla\left(\varepsilon \chi^{j, h}\left(x_{k}, x / \varepsilon\right)\right) \frac{\partial \bar{u}^{H}}{\partial x_{j}}
\end{aligned}
$$

and we obtain

$$
\begin{aligned}
& \sum_{K \in \mathcal{T}_{H}}\left\|\nabla\left(u_{p}^{\varepsilon}-\bar{u}_{p}^{\varepsilon}\right)\right\|_{L^{2}(K)}^{2} \leq \sum_{K \in \mathcal{T}_{H}}\left\|\nabla\left(u^{H}-\bar{u}^{H}\right)\right\|_{L^{2}(K)}^{2} \\
& +\sum_{K \in \mathcal{T}_{H}}\left\|\sum_{j=1}^{n} \nabla\left(\varepsilon \chi^{j}\left(x_{k}, x / \varepsilon\right)\right)\left(\frac{\partial u^{H}}{\partial x_{j}}-\frac{\partial \bar{u}^{H}}{\partial x_{j}}\right)\right\|_{L^{2}(K)}^{2} \\
& +\sum_{K \in \mathcal{T}_{H}}\left\|\sum_{j=1}^{n} \nabla\left(\varepsilon\left(\chi^{j}-\chi^{j, h}\right)\left(x_{k}, x / \varepsilon\right)\right) \frac{\partial \bar{u}^{H}}{\partial x_{j}}\right\|_{L^{2}(K)}^{2}
\end{aligned}
$$

The first two terms are bounded by $\left(C M^{-\frac{2}{n}}\|f\|_{L^{2}(\Omega)}\right)^{2}$ using (3.9) and (3.15). For the last term, we take a finite covering of $K \subset \bigcup_{x_{l} \in K} K_{\varepsilon}\left(x_{l}\right)$, where $K_{\varepsilon}\left(x_{l}\right)=$ $x_{l}+\varepsilon[-1 / 2,1 / 2]^{n}$, and using (3.14) we bound it by $\left(C M^{-\frac{1}{n}}\left\|\nabla \bar{u}^{H}\right\|_{L^{2}(\Omega)}\right)^{2}$. Using the norm defined in (3.25) and (3.6) we obtain $\left\|u_{p}^{\varepsilon}-\bar{u}_{p}^{\varepsilon}\right\|_{\bar{H}^{1}(\Omega)} \leq C M^{-\frac{1}{n}}\|f\|_{L^{2}(\Omega)}$. Using (2.12) and the triangle inequality completes the proof.

4. Numerical experiments. We present in this section numerical experiments which confirm our theoretical estimates and show that the obtained bounds are sharp. Therefore, we consider the simple following (quasi-one-dimensional) model problem:

$$
\begin{array}{rlrl}
-\nabla \cdot\left(a\left(\frac{x}{\varepsilon}\right) \nabla u^{\varepsilon}\right) & =f(x) \quad \text { in } \Omega & =(0,1)^{2}, \\
\left.u^{\varepsilon}\right|_{\Gamma_{D}} & =0 & & \text { on } \Gamma_{D}:=\left\{x_{1}=0\right\} \cup\left\{x_{1}=1\right\}, \\
\left.n \cdot\left(a\left(\frac{x}{\varepsilon}\right) \nabla u^{\varepsilon}\right)\right|_{\Gamma_{N}} & =0 & & \text { on } \Gamma_{N}:=\partial \Omega \backslash \Gamma_{N},
\end{array}
$$


where $a(y)=\left(\cos 2 \pi y_{1}+2\right), y=\left(y_{1}, y_{2}\right) \in Y=(0,1)^{2}$, and $f(x) \equiv 1$. The exact solution, as well as the homogenized tensor, can be derived analytically:

$$
u^{\varepsilon}=-\int_{0}^{x_{1}} \frac{t}{a(t / \varepsilon)} d t+\frac{\int_{0}^{1} \frac{t}{a(t / \varepsilon)} d t}{\int_{0}^{1} \frac{1}{a(t / \varepsilon) d t}} \int_{0}^{x_{1}} \frac{1}{a(t / \varepsilon)} d t, \quad a^{0}=\left(\begin{array}{cc}
\left(\int_{0}^{1} \frac{1}{a\left(y_{1}\right)} d y_{1}\right)^{-1} & 0 \\
0 & 2
\end{array}\right) .
$$

Therefore, we can compute a reference solution for the fine-scale solution and for the homogenized solution with high precision. The reference solution for $u^{\varepsilon}$ is computed with the above integral formula (with a very precise numerical integration scheme). The homogenized solution is a quadratic polynomial obtained from the solution of (4.1) with $a^{0}$ instead of $a^{\varepsilon}$ and can be easily computed. For the implementation of the FE-HMM, we enforced periodically the coupling between micro- and macroscales (the periodic boundary conditions) through Lagrange multipliers as proposed in [2].

In the figures below, we denote $h_{M} \simeq M^{-\frac{1}{2}}$, where $M$ is the number of DOF of the micro FE space $S_{p e r}^{1}\left(K_{\varepsilon}\right)$ defined in (3.1). If we denote by $h$ the mesh size of this micro FE space, we have $h=\varepsilon h_{M}$ (see Remark 3.4). We also denote the macromesh size by $H \simeq N^{-\frac{1}{2}}$, where $N$ is the number of DOF of the macro FE space $S_{0}^{1}\left(\Omega, \mathcal{T}_{H}\right)$ defined in (2.4). We compute for a macromesh refinement $H=(1 / 2)^{d}$, $d=1, \ldots, 6$, the solutions of the FE-HMM with several scaled micromesh sizes: $h_{M}=1 / 10,1 / 20,1 / 40,1 / 80,1 / 100$. Let us discuss the different experiments and results.

Convergence results for the $L^{2}$ norm. We see in Figure 4.1 (left) that for a given micromesh size, the error decreases quadratically with the macromesh size until it reaches $H \simeq h_{M}$ in agreement with Corollary 3.7. To further decrease the error, the micromesh has to be refined. The convergence is independent of $\varepsilon$ (see Corollary 3.7), and we present the result for $\varepsilon=10^{-1}$. (The cell problems are solved with a microtriangulation $\mathcal{T}_{h}$, which is an $\varepsilon$ scaled mesh of a triangulation of the unit-size cell.)

We next compare the fine-scale solution of problem (4.1)-(4.3) and the solution of the FE-HMM. We see in Figure 4.2 that there is now a dependency towards $\varepsilon$ as predicted by Corollary 3.7. When the error is dominated by $\varepsilon$, a refinement of the micromesh does not improve the result (see Figure 4.2 (left)). If we decrease $\varepsilon$ from $10^{-1}$ to $10^{-3}$, we see in Figure 4.2 (right) that the error is now dominated by the microproblem, and a micromesh refinement improves the result as predicted by Corollary 3.7.

Convergence results for the $H^{1}$ norm. We see in Figure 4.1 (right) that for a given micromesh size, the error decreases linearly with the macromesh size until it reaches $H \simeq h_{M}$, i.e., $N \simeq M$, which is more severe than predicted by Theorem 3.6. This may be because of a bigger error constant for the microscale error (3.10) than for the macroscale semidiscrete error (2.14). To further decrease the error, the micromesh has to be refined. The convergence is again independent of $\varepsilon$ (see Theorem 3.6), and we present the results for $\varepsilon=10^{-1}$.

Convergence for noninteger cell size. We have discussed in section 2.1 that the FE-HMM is not restricted to problems with periodic microstructures. In this situation, the optimal size of the sampling domains may not be known. In the following, we apply the FE-HMM to the problem (4.1) but with cell size $K_{\delta}$ instead of $K_{\varepsilon}$, with $\delta / \varepsilon \notin \mathbb{N}$. In this case, it has been shown that $\left\|u^{0}-u^{H}\right\|_{L^{2}(\Omega)} \leq C\left(H+\frac{\varepsilon}{\delta}+\delta\right)$, where $u^{H}$ is the (semidiscrete) solution of (2.9) with the cell problem $(2.8)$ in $H^{1}\left(K_{\delta}\right)$, with Dirichlet boundary conditions (see section 2.1). A fine-scale error as in (3.15) should 

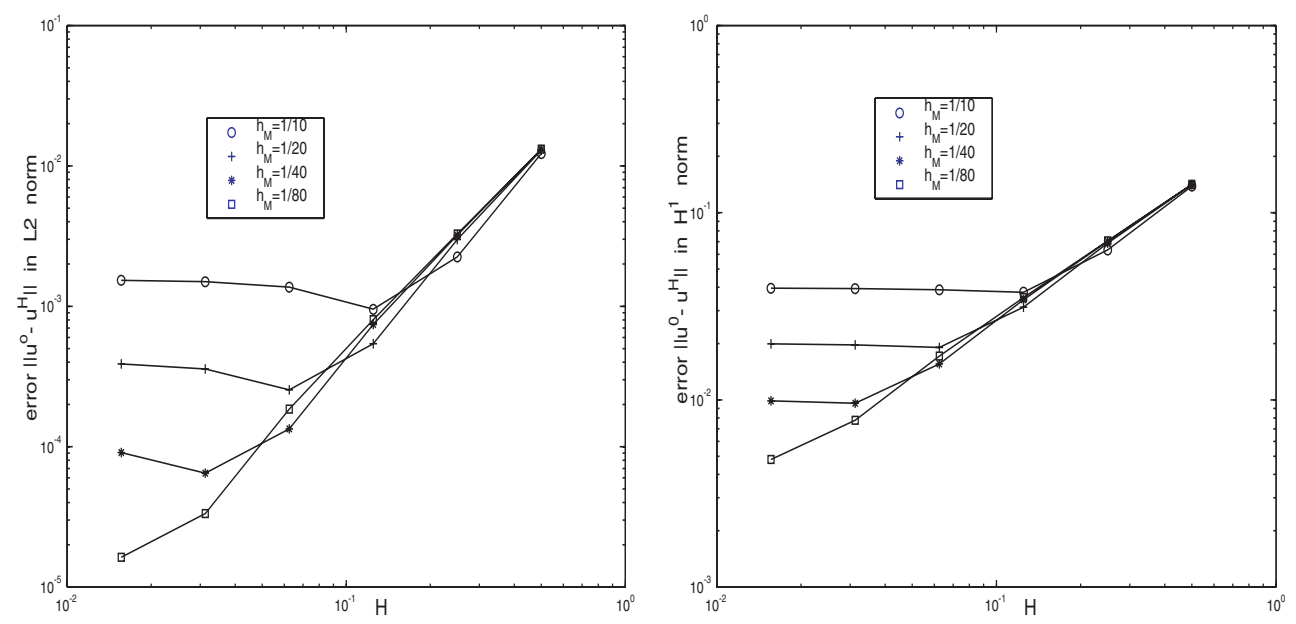

FIG. 4.1. Convergence rate of the error between the macrosolution of the FE-HMM for problem (4.1)-(4.3), with $\varepsilon=10^{-1}$ and decreasing micromeshes, and the homogenized solution of problem $(4.1)-(4.3)$.
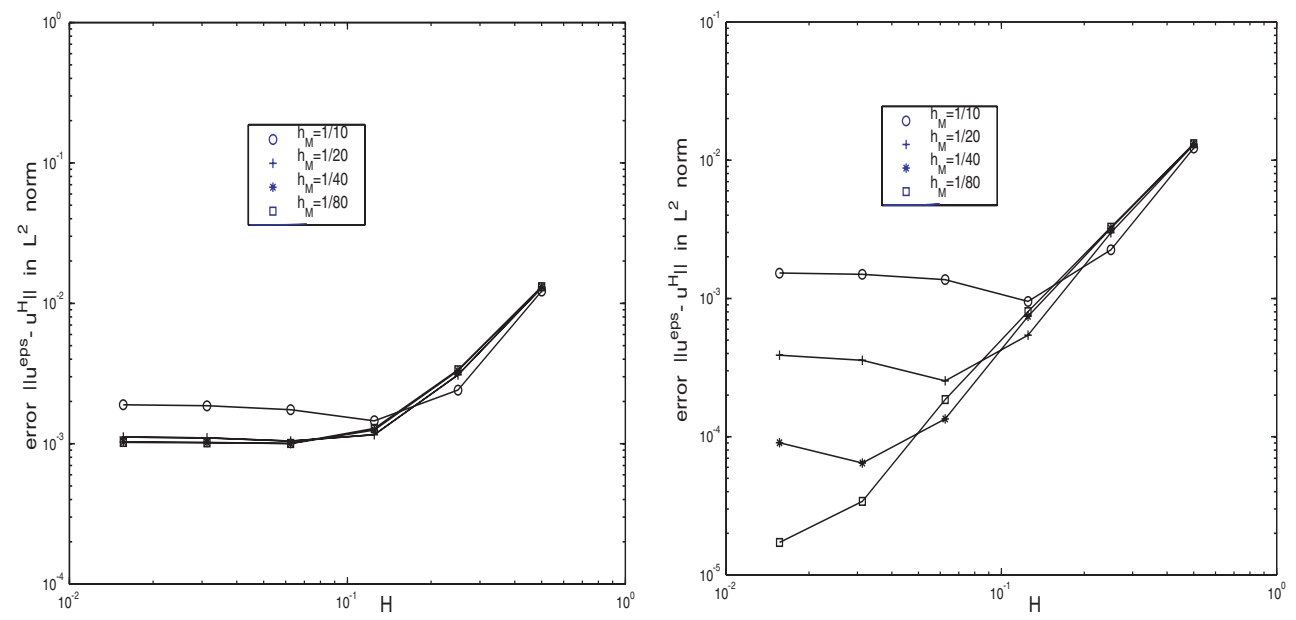

FIG. 4.2. Convergence rate of the error between the macrosolution of the FE-HMM with decreasing micromeshes and the fine-scale solution of problem (4.1)-(4.3), with $\varepsilon=10^{-1}$ (left) and $\varepsilon=10^{-3}$ (right).

also appear in the fully discrete analysis. In this case, however, the remainder error terms $\frac{\varepsilon}{\delta}+\delta$ can dominate, in some situation, the error of the microsolver. For the first experiment, we take $\varepsilon=10^{-3}$ and a noninteger ratio $\frac{\delta}{\varepsilon}=79 / 32$ for the cell size. We adapt the value of $h_{M}$ to have the same number of sampling points per period $\varepsilon$ as before.

We see in Figure 4.3 (left) that the error first decreases (according to the above estimate) and becomes stationary, indicating that the remainder error terms $\frac{\varepsilon}{\delta}+\delta$ may dominate the global error and a refinement of the micromesh does not have any impact. In order to minimize the influence of these terms, let us choose the theoretical best value for $\delta=\sqrt{\varepsilon}$. In this case, we see in Figure 4.3 (right) that the error in the 

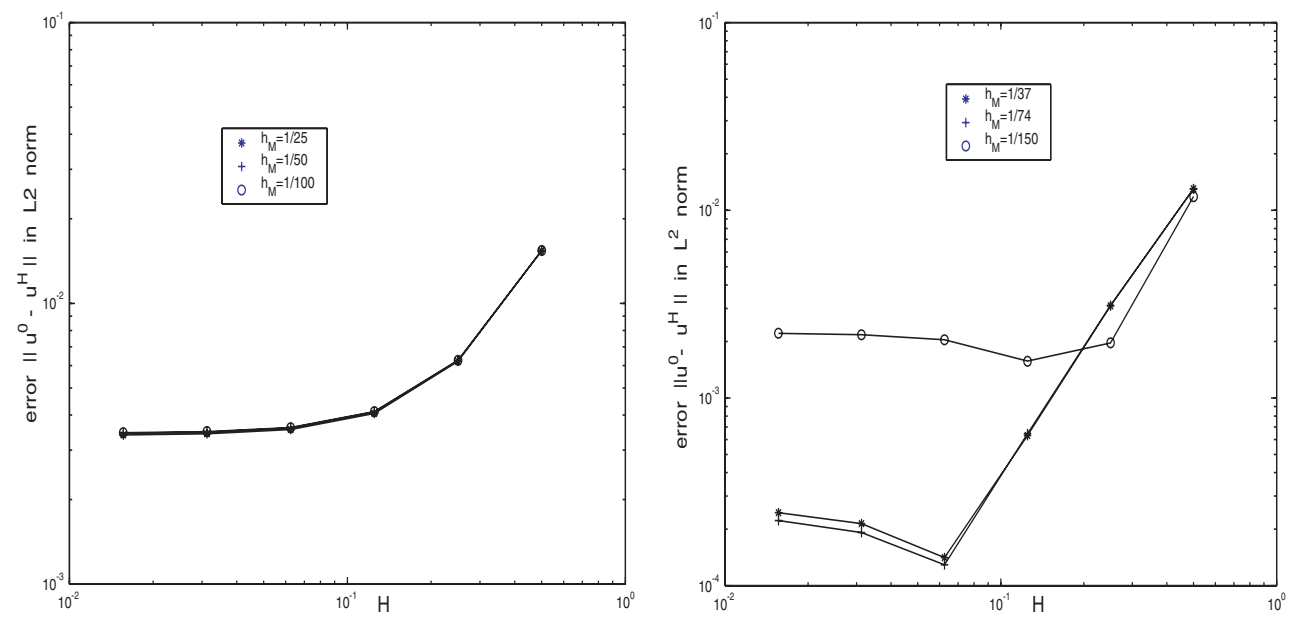

FIG. 4.3. Convergence rate of the error between the macrosolution of the FE-HMM, with cell size $K_{\delta}$ and decreasing micromeshes, and the fine-scale solution of problem (4.1)-(4.3), with $\varepsilon=10^{-3}, \delta=79 / 32 \cdot \varepsilon$ (left) and $\delta=\sqrt{\varepsilon}$ (right).

microsolver begins to dominate when $H$ decreases, and a refinement of the micromesh improves the results, as predicted by Corollary 3.7 in the case $K_{\delta}=K_{\varepsilon}$, until the remainder terms again dominate.

Acknowledgments. The author is grateful to Christoph Schwab for helpful discussions and to the referees, whose suggestions and comments improved the paper.

\section{REFERENCES}

[1] A. Abdulle and W. E, Finite difference HMM for homogenization problem, J. Comput. Phys., 191 (2003), pp. 18-39.

[2] A. Abdulle And C. Schwab, Heterogeneous multiscale FEM for diffusion problems on rough surfaces, Multiscale Model. Simul., 3 (2005), pp. 195-220.

[3] A. Bensoussan, J.-L. Lions, and G. Papanicolaou, Asymptotic Analysis for Periodic Structures, North-Holland, Amsterdam, 1978.

[4] D. Cioranescu and P. Donato, An Introduction to Homogenization, Oxford University Press, New York, 1999.

[5] W. E, P. Ming, And P. Zhang, Analysis of the heterogeneous multi-scale method for elliptic homogenization problems, J. Amer. Math. Soc., 18 (2005), pp. 121-156.

[6] W. E And B. Engquist, The heterogeneous multiscale methods, Commun. Math. Sci., 1 (2003), pp. $87-132$.

[7] W. E, X. Li, ANd E. VANDEn-EiJden, Some recent progress in multiscale modeling, in Multiscale Modelling and Simulation, Lect. Notes Comput. Sci. Eng. 39, Springer, Berlin, 2004, pp. $3-22$.

[8] B. Engquist and O. Runborg, Wavelet-based numerical homogenization with applications, in Multiscale and Multiresolution Methods, Lect. Notes Comput. Sci. Eng. 20, Springer, Berlin, 2002, pp. 97-148.

[9] T.-Y. Hou, X.-H. Wu, AND Z. CAI, Convergence of a multi-scale finite element method for elliptic problems with rapidly oscillating coefficients, Math. Comp., 68 (1999), pp. 913-943.

[10] V. V. Jikov, S. M. Kozlov, And O. A. Oleinik, Homogenization of Differential Operators and Integral Functionals, Springer, Berlin, Heidelberg, 1994.

[11] A. M. Matache And C. Schwab, Two-scale FEM for homogenization problems, M2AN Math. Model. Numer. Anal., 36 (2002), pp. 537-572.

[12] P. Ming And P. Zhang, Analysis of the Heterogeneous Multi-scale Method for Parabolic Homogenization Problems, preprint, 2003, http://www.math.princeton.edu/multiscale/. 
[13] P. Ming and X.-Y. Yue, Numerical Methods for Multiscale Problems, preprint, 2003, http:// www.math.princeton.edu/multiscale/.

[14] S. Moskow AND M. Vogelius, First order corrections to the homogenized eigenvalues of a periodic composite medium, Proc. Roy. Soc. Edinburgh Sect. A, 127 (1997), pp. 1263-1299.

[15] N. Neuss, W. JäGer, And G. Wittum, Homogenization and multigrid, Computing, 66 (2001), pp. $1-26$.

[16] J. T. Oden and K. S. Vemaganti, Estimation of local modeling error and global-oriented adaptive modeling of heterogeneous materials: Error estimates and adaptive algorithms, J. Comput. Phys., 164 (2000), pp. 22-47. 